\title{
Response
}

\section{Efficacy and Safety of Voglibose Plus Metformin in Patients with Type 2 Diabetes Mellitus: A Randomized Controlled Trial (Diabetes metab J 2019;43;276-86)}

\author{
Tae Jung $\mathrm{Oh}^{1}$, Sung Hee $\mathrm{Choi}^{1,2}$ \\ ${ }^{1}$ Department of Internal Medicine, Seoul National University Bundang Hospital, Seoul National University College of Medicine, Seongnam, \\ ${ }^{2}$ Department of Internal Medicine, Seoul National University College of Medicine, Seoul, Korea
}

We appreciate Dr. Seok and Dr. Sohn for their thoughtful comments on our recently published article, "Efficacy and safety of voglibose plus metformin in patients with type 2 diabetes mellitus: a randomized controlled trial" [1]. They pointed out the potential role of fixed dose combination of voglibose and metformin (vogmet) in patients with type 2 diabetes mellitus with higher initial hyperglycemia. Also, this medication would be more efficacious in Korean population consuming carbohydrate-rich diet, considering the mode of action of alpha-glucosidase inhibitor that prohibits the rapid absorption of glucose.

We want to emphasize the data of gastrointestinal adverse events (AEs) and body weight reduction in our study, which was discussed by Dr. Seok and Dr. Sohn. First, we thought that lower dose of metformin might be the contributing factor of lower incidence of gastrointestinal AEs in vogmet (fixed dose combination) group compared with metformin group. However, the mechanism of metformin-associated gastrointestinal AEs has still not been well studied, and it might be related with individual genetic factors [2]. In this study, although we do not know the exact mechanism, voglibose at least did not increase the gastrointestinal AEs when it was used with low dose metformin. Second, a previous meta-analysis study [3] demonstrated body weight reduction effect of alpha-glucosidase inhibitors compared with placebo (weighted mean difference, $-1.0 \mathrm{~kg} ; 95 \%$ confidence interval, -1.69 to $-0.31 \mathrm{~kg}$ ) in Asian as well as Caucasian. Therefore, some studies showed weightneutrality or weight-benefit, but this finding is not globally acceptable. In fact, our study showed a much more significant body weight reduction in vogmet group than metformin group $(-1.63 \mathrm{~kg}$ vs. $-0.86 \mathrm{~kg}, P<0.05)$. Based on our study and others, it will be interesting to select appropriate patients who will benefit in terms of both reducing hyperglycemia and body weight.

We totally agree with their opinion that measuring glucose fluctuation using continuous glucose monitoring system (CGMS) will provide more valuable understanding for our study. Another alpha-glucosidase inhibitor, miglitol has been tested whether it reduced glucose variability in patients with acute coronary syndrome [4]. This study showed that miglitol reduced standard deviation and mean amplitude of glycemic excursion compared with pretreatment. Further study is necessary to test whether vogmet reduces glucose variability in outpatient setting using CGMS, but we can assume that vogmet will have similar benefit as miglitol in glucose variability due to its composition and nature. We also think that it will be necessary to test body composition and other cardiovascular risk factors such as blood pressure, lipid profiles, and insulin resistance, to understand the possible cardiovascular benefits of vogmet in future.
Corresponding author: Sung Hee Choi (iD https://orcid.org/0000-0003-0740-8116 Department of Internal Medicine, Seoul National University Bundang Hospital, Seoul National University College of Medicine, 82 Gumi-ro 173beon-gil, Bundang-gu,

Seongnam 13620, Korea

E-mail: drshchoi@snu.ac.kr
This is an Open Access article distributed under the terms of the Creative Commons Attribution Non-Commercial License (http://creativecommons.org/licenses/by-nc/4.0/) which permits unrestricted non-commercial use, distribution, and reproduction in any medium, provided the original work is properly cited. 


\section{CONFLICTS OF INTEREST}

No potential conflict of interest relevant to this article was reported.

\section{REFERENCES}

1. Oh TJ, Yu JM, Min KW, Son HS, Lee MK, Yoon KH, Song YD, Park JY, Jeong IK, Cha BS, Kim YS, Baik SH, Kim IJ, Kim DM, Kim SR, Lee KW, Park JH, Lee IK, Park TS, Choi SH, Park SW. Efficacy and safety of voglibose plus metformin in patients with type 2 diabetes mellitus: a randomized controlled trial. Diabetes Metab J 2019;43:276-86.

2. McCreight LJ, Bailey CJ, Pearson ER. Metformin and the gas- trointestinal tract. Diabetologia 2016;59:426-35.

3. Cai X, Han X, Luo Y, Ji L. Comparisons of the efficacy of alpha glucosidase inhibitors on type 2 diabetes patients between Asian and Caucasian. PLoS One 2013;8:e79421.

4. Shimabukuro M, Tanaka A, Sata M, Dai K, Shibata Y, Inoue Y, Ikenaga $\mathrm{H}$, Kishimoto S, Ogasawara K, Takashima A, Niki T, Arasaki O, Oshiro K, Mori Y, Ishihara M, Node K; Collaborators on the Effect of Miglitol on Glucose Metabolism in Acute Coronary Syndrome (MACS) Study. a-Glucosidase inhibitor miglitol attenuates glucose fluctuation, heart rate variability and sympathetic activity in patients with type 2 diabetes and acute coronary syndrome: a multicenter randomized controlled (MACS) study. Cardiovasc Diabetol 2017;16:86. 